\title{
Questionnaire in English
}

\section{Cardio Metabolic Risk}

Date: I__ I I I I

Diabetes: $\square$ yes $\square$ no $\begin{gathered}\text { if yes T2D : } \square \text { yes } \square \text { no } \\ \text { Diabetes vintage: / }\end{gathered}$

\section{Demographic Parameters}

Number: /

Group: /_ / $\quad($ Case $=1 ;$ Control $=0) \quad($ case $=$ History of CAD or Stroke $)$

Gender: $\square$ Male $\quad \square$ Female $\quad(1=\mathrm{M} ; 2=\mathrm{F})$

Birth date: I__ l_ _ I _ I

Place of birth:

ZIP code of residence:

\section{History related to cardiovascular and arterial diseases.}

\section{Family history}

Fam hist for hypertension: $\square$ yes $\square$ no

Fam hist for diabetes: $\square$ yes $\square$ no

\section{Personal}

Hypertension: $\square$ yes $\square$ no

Stroke: $\square$ yes $\square$ no

CAD: $\square$ yes $\square$ no

Myocardial Infarction : $\square$ yes $\square$ no

Angina: $\square$ yes $\square$ no

Lower limbs arteriopathy: $\square$ yes $\square$ no $\quad$ if yes, Amputation : $\square$ yes $\square$ no

\section{Mode de vie}

Tobacco: $\square$ yes $\square$ no (yes = current smoker or stop for less than 10 years, no = others)

Alcohol: $\square$ yes $\square$ no

If yes: wine $\square$ yes $\square$ no; rum: $\square$ yes $\square$ no; nbr glasses / day / _ / 
Sports: $\square$ yes $\square$ no; if yes nbr hours weekly / _ /

\section{Current treatment}

Antihypertensive: $\square$ yes $\square$ no

ACE: $\square$ yes $\square$ no $\quad$ Calcium blocker : $\square$ yes $\square$ no

Beta blocker: $\square$ yes $\square$ no other Tt : $\square$ yes $\square$ no $\quad$ if yes :

Anti-diabetic Tt: $\square$ yes $\square$ no

If yes $\square$ OAD $\square$ Insulin $\square$ both

Hypolipemic Tt: $\square$ yes $\square$ no

Antiplatelet agents: $\square$ yes $\square$ no

Clinical data

Weight:/ /kg Height: / $/ \mathrm{m}$

Waist circumference / $/ \mathrm{cm}$

\section{$\underline{\text { Electrocardiogram }}$}

ECG: $\quad \square$ normal $\square$ abnormalities

If abnormal: ischemic $\square$ yes $\square$ no

\section{$\underline{\text { Biological data }}$}

C-RP:

$\mathrm{mg} / \mathrm{L}$

Hemoglobin: / _ / g/dl

Creatinine: I__ $/ \mu \mathrm{mol} / \mathrm{L}$

Hématocrite: $\quad / \quad / \%$

Total Cholesterol: /_ _ $/ \mathrm{mmol} / \mathrm{L}$

HDL-Cholesterol: /_ _ $\mathrm{mmol} / \mathrm{L}$

LDL-Cholesterol: /_ _ / $\mathrm{mmol} / \mathrm{L}$

Triglycerides: / _ _ $\mathrm{mmol} / \mathrm{L}$

FBG: /__ / $/ \quad \mathrm{mmol} / \mathrm{L}$ 